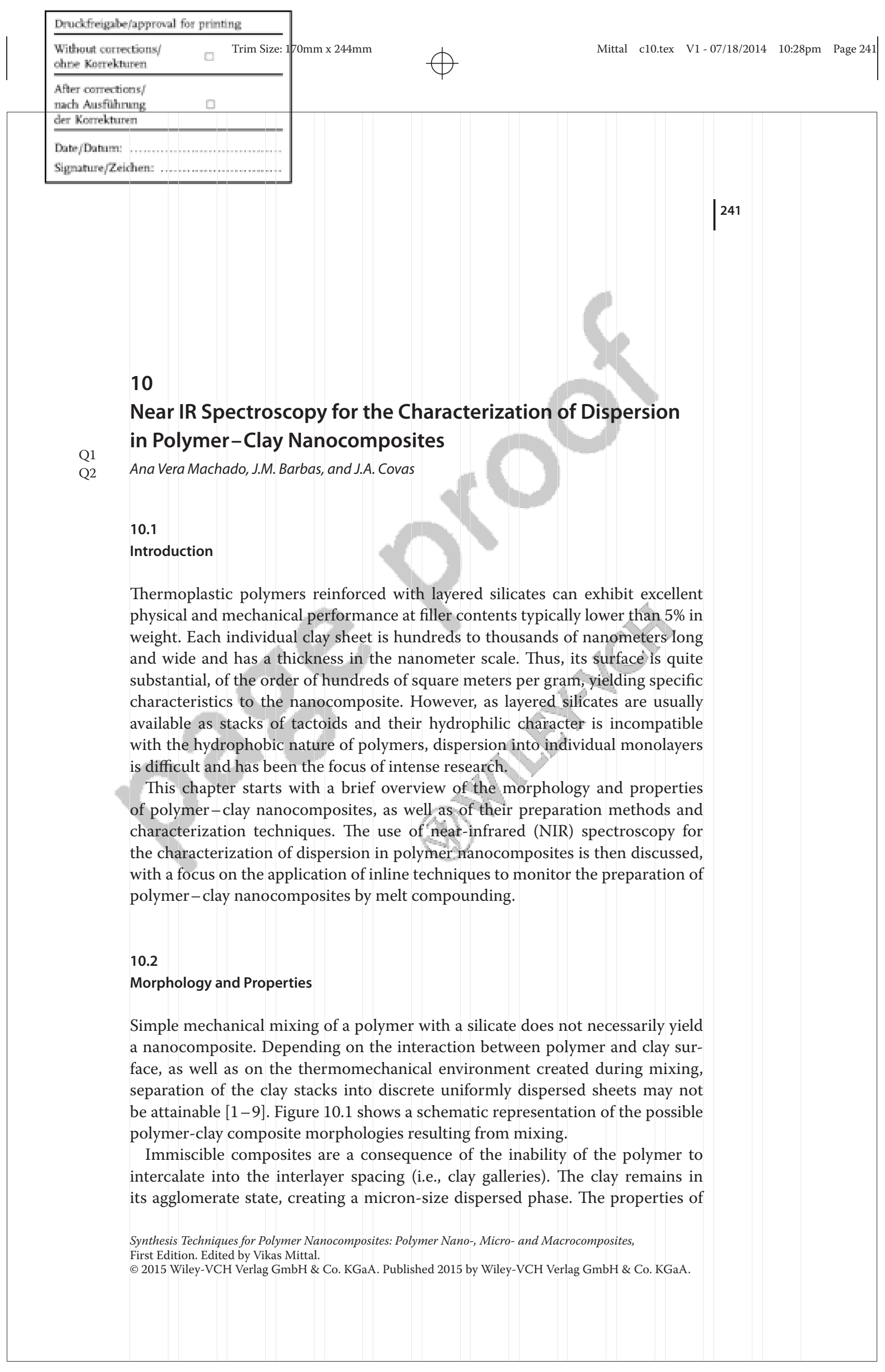

\title{
Near IR Spectroscopy for the Characterization of Dispersion in Polymer-Clay Nanocomposites
}

10.1

Thermoplastic polymers reinforced with layered silicates can exhibit excellent physical and mechanical performance at filler contents typically lower than $5 \%$ in weight. Each individual clay sheet is hundreds to thousands of nanometers long and wide and has a thickness in the nanometer scale. Thus, its surface is quite substantial, of the order of hundreds of square meters per gram, yielding specific characteristics to the nanocomposite. However, as layered silicates are usually available as stacks of tactoids and their hydrophilic character is incompatible ith the hydrophobic nature of polymers, dispersion into individual monolayers difficult and has been the focus of intense research.

This chapter starts with a brief overview of the morphology and properties of polymer-clay nanocomposites, as well as of their preparation methods and haracterization techniques. The use of near-infrared (NIR) spectroscopy for the characterization of dispersion in polymer nanocomposites is then discussed, ith a focus on the application of inline techniques to monitor the preparation of polymer-clay nanocomposites by melt compounding.

\section{2}

Simple mechanical mixing of a polymer with a silicate does not necessarily yield a nanocomposite. Depending on the interaction between polymer and clay surface, as well as on the thermomechanical environment created during mixing, lymer-clay composite morphologies resulting from mixing.

Immiscible composites are a consequence of the inability of the polymer to ntercalate into the interlayer spacing (i.e., clay galleries). The clay remains in

Synthesis Techniques for Polymer Nanocomposites: Polymer Nano-, Micro- and Macrocomposites, irst Edition. Edited by Vikas Mittal.

2015 Wiley-VCH Verlag GmbH \& Co. KGaA. Published 2015 by Wiley-VCH Verlag GmbH \& Co. KGaA 


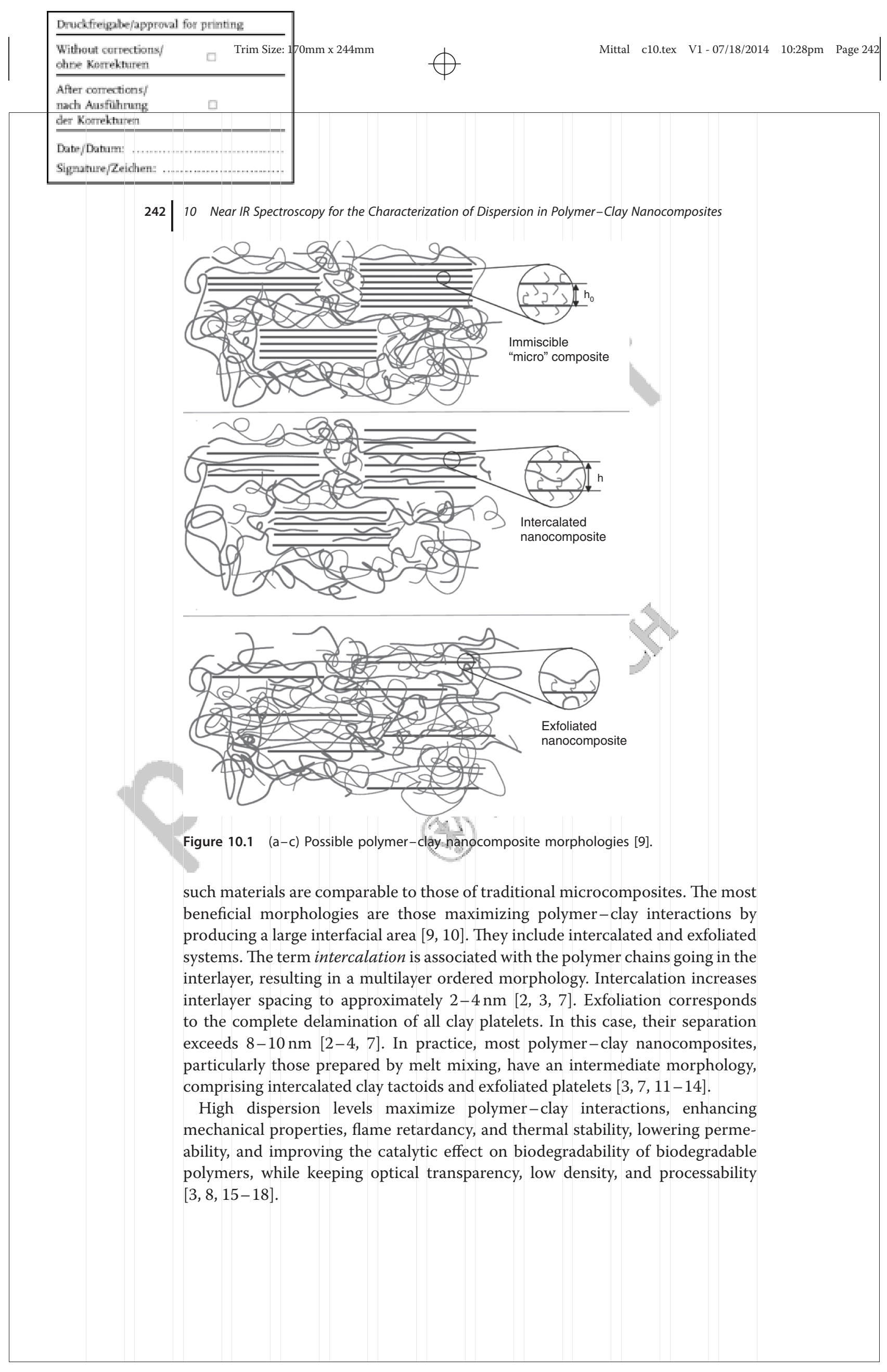




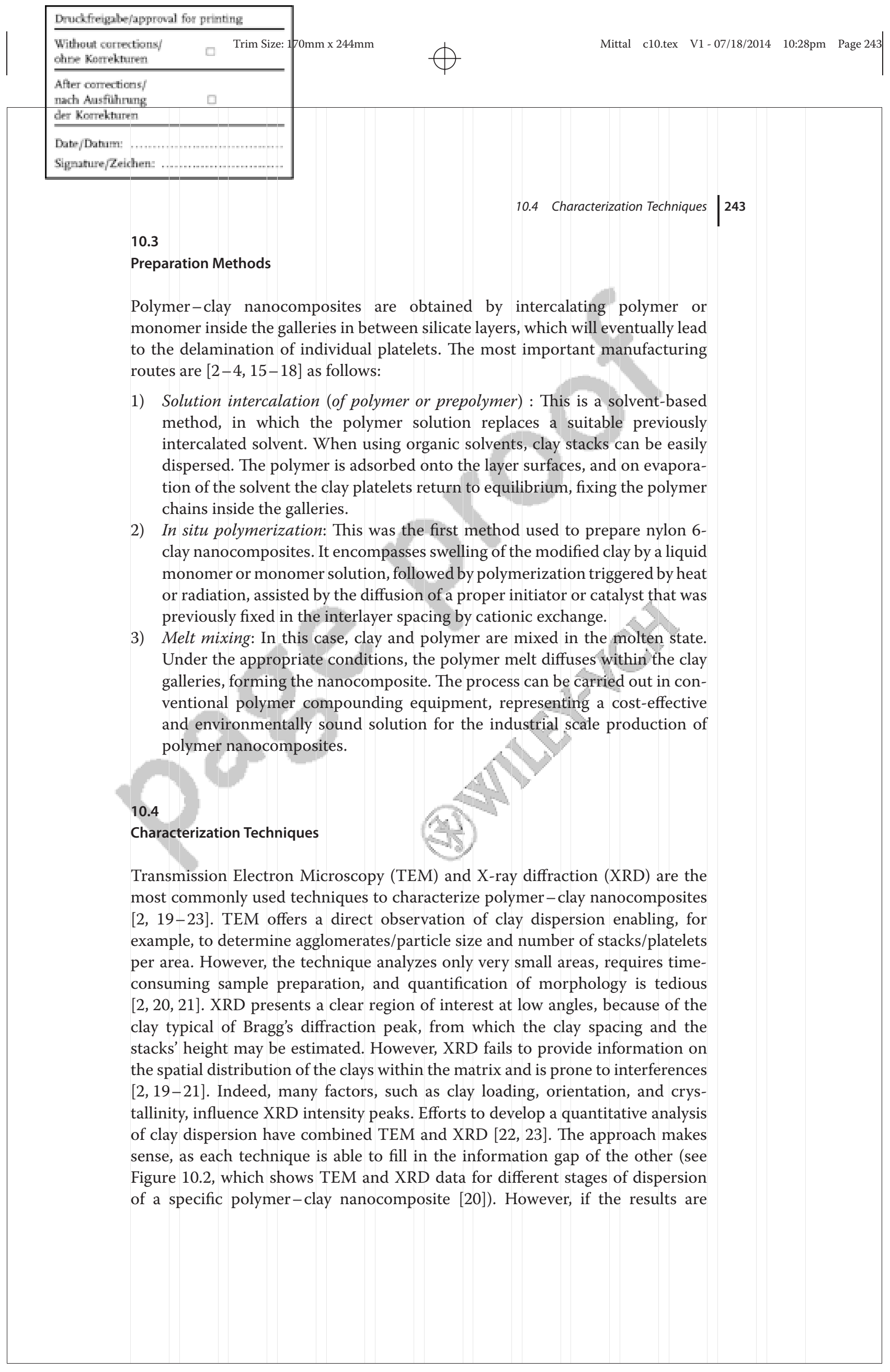




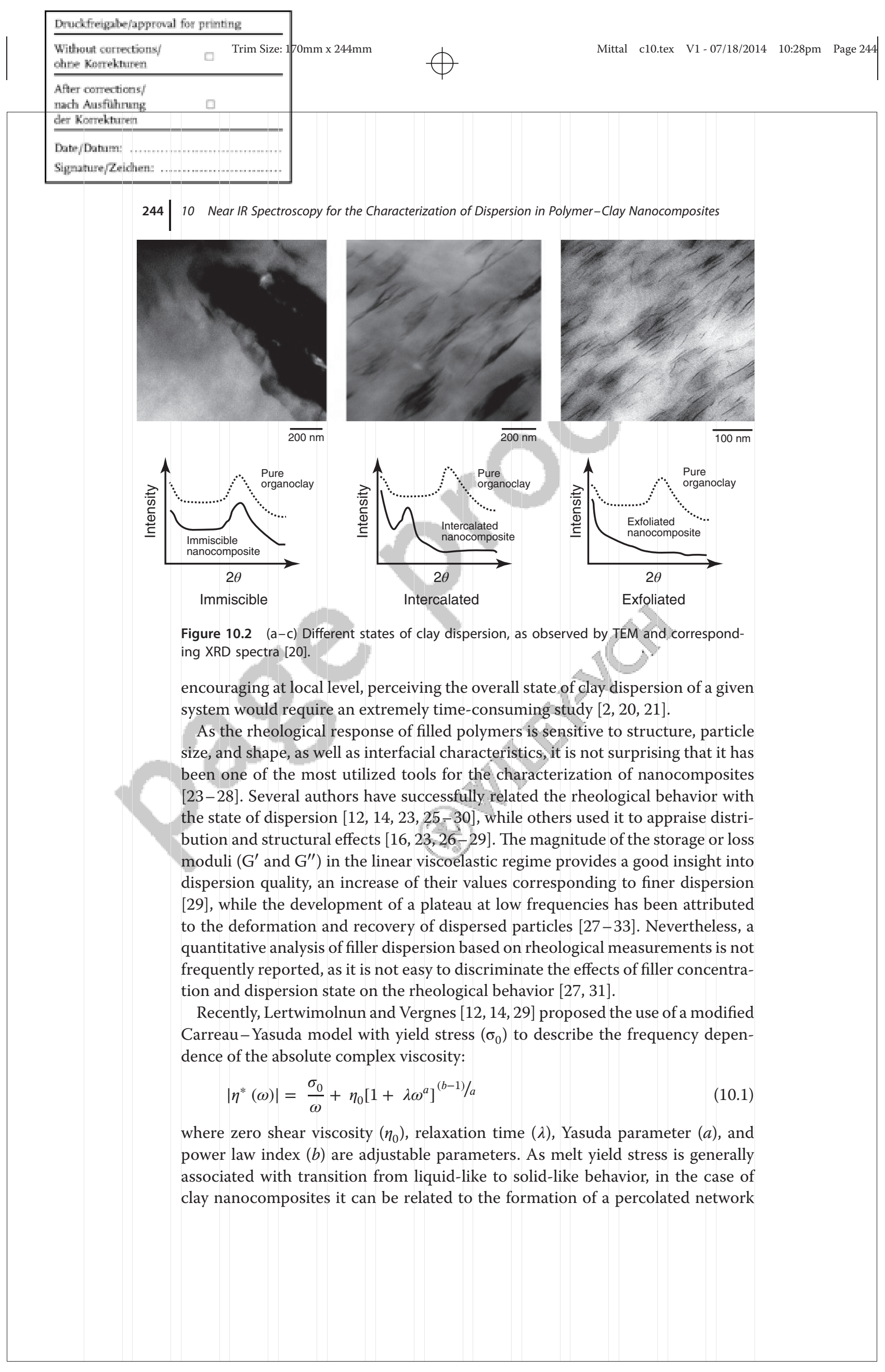




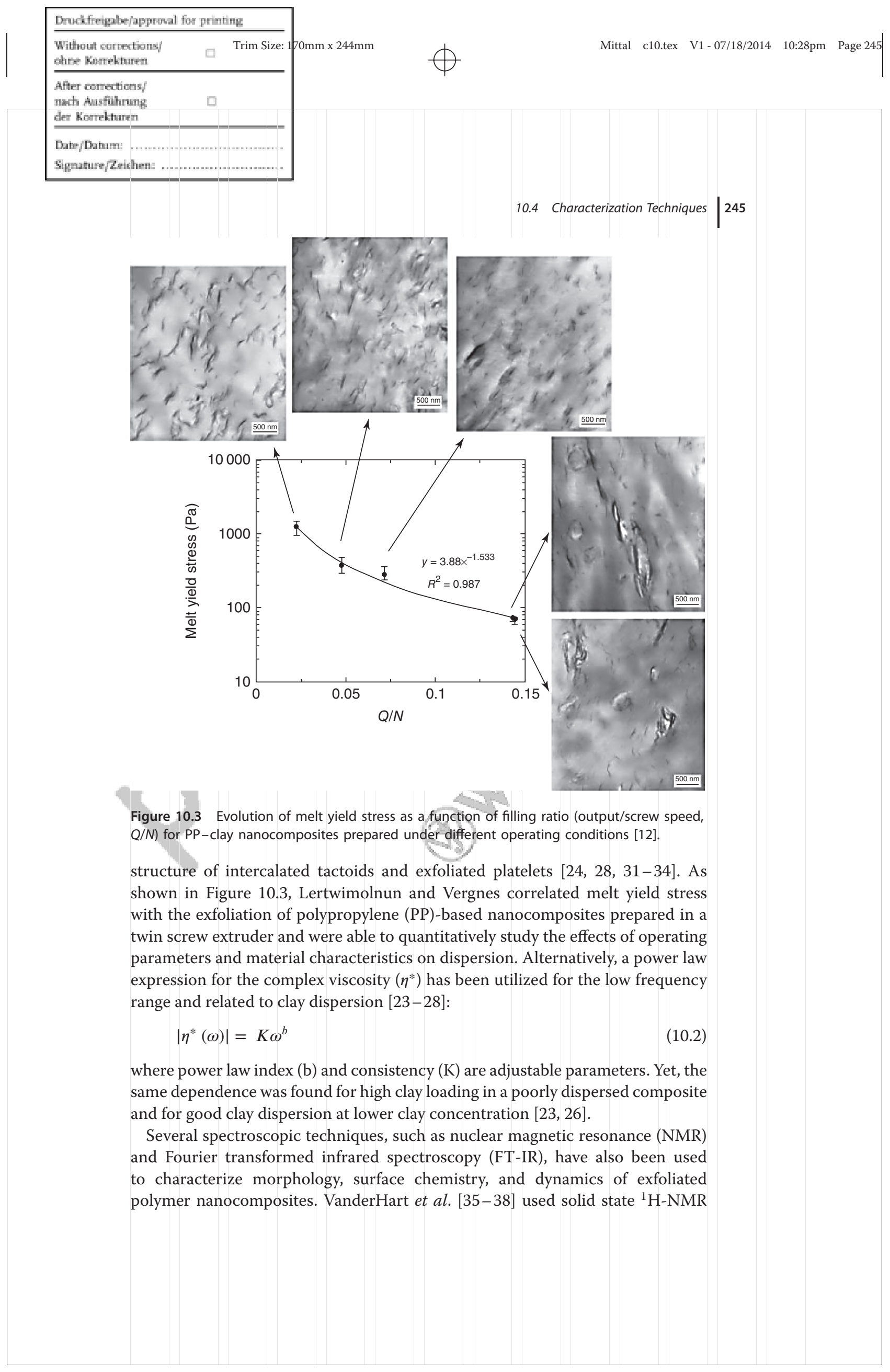




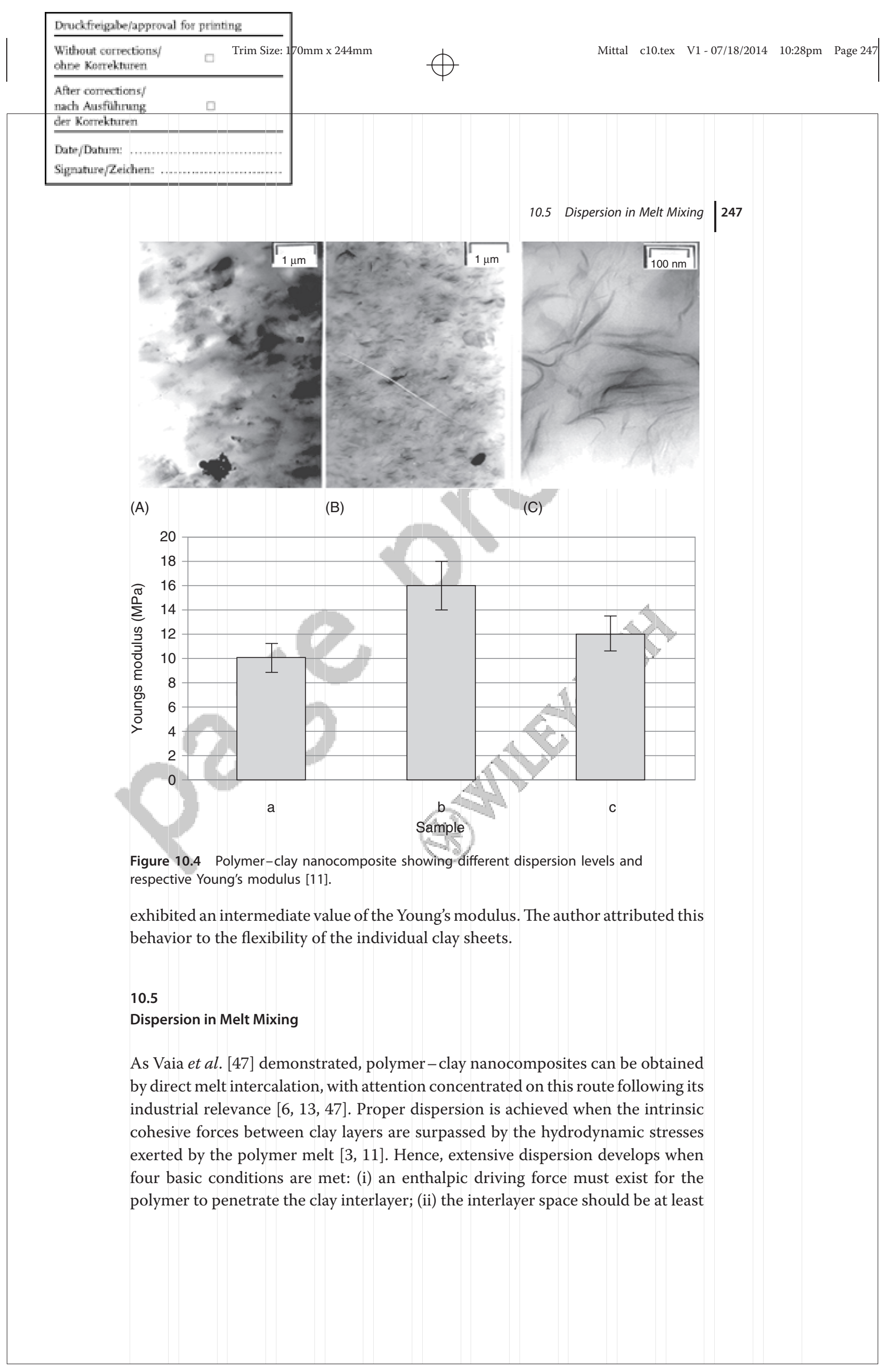




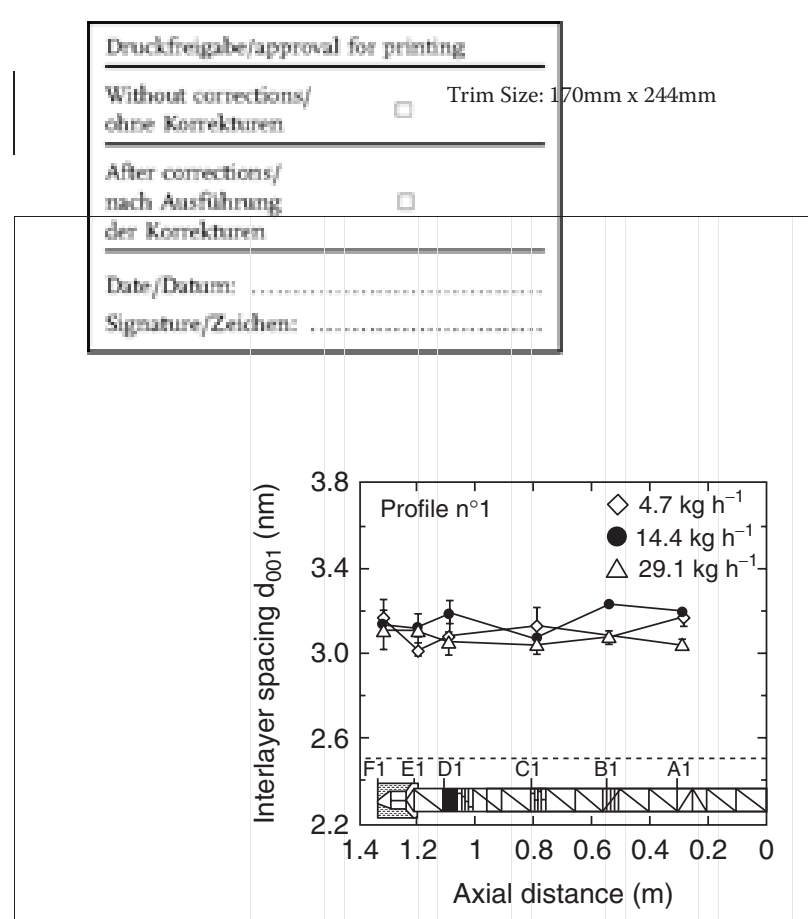

Mittal c10.tex V1-07/18/2014 10:28pm Page 249

10.6 Online and Inline Monitoring of Dispersion

Figure 10.5 Effect of feed rate on the variation along the extruder of interlayer spacing (a) and melt yield stress (b) [14].

mild shearing conditions (or in a medium to low viscosity matrix) applied during sufficient time, whereas extensive exfoliation requires a high level of shearing/deformation.

Most of the above studies involved small-scale experiments [11, 49, 52] and/or the characterization of samples collected after completing mixing [29], for example, at the extrusion die exit $[7,13]$. The evolution of clay dispersion along an extruder, where a complex nonisothermal 3D flow develops, seems to be less well understood. Lertwimolnun and Vergnes [12, 14] characterized postmortem samples collected from various locations along the axis of a corotating twin screw extruder (TSE). They concluded that both intercalation and exfoliation can reach relatively high levels immediately after melting. They also observed that less restrictive screw profiles yielded better dispersion levels. Furthermore, depending on the combination of screw profile and operating conditions, these authors observed an apparent reversion of dispersion evolution along the screw. This is illustrated in Figure 10.5 for the effect of feed rate. The graph on the left presents the evolution of the interlayer spacing $\left(\mathrm{d}_{001}\right)$, as determined by XRD, which is associated with intercalation. The plot on the right presents the progress of melt yield stress, which is linked to exfoliation.

10.6

\section{Online and Inline Monitoring of Dispersion}

During practical compounding and processing, the parameters that are continuously monitored (typically temperature, melt pressure, and motor torque) do not provide sufficient information on the characteristics of the system being processed. Thus, the possibility of assessing in real time the dispersion of a nanocomposite on processing is an important scientific and technological target, as it can be used to assist the definition of material recipes, the optimization of operating conditions and/or screw design, as well as for quality control and, ultimately, process control. 


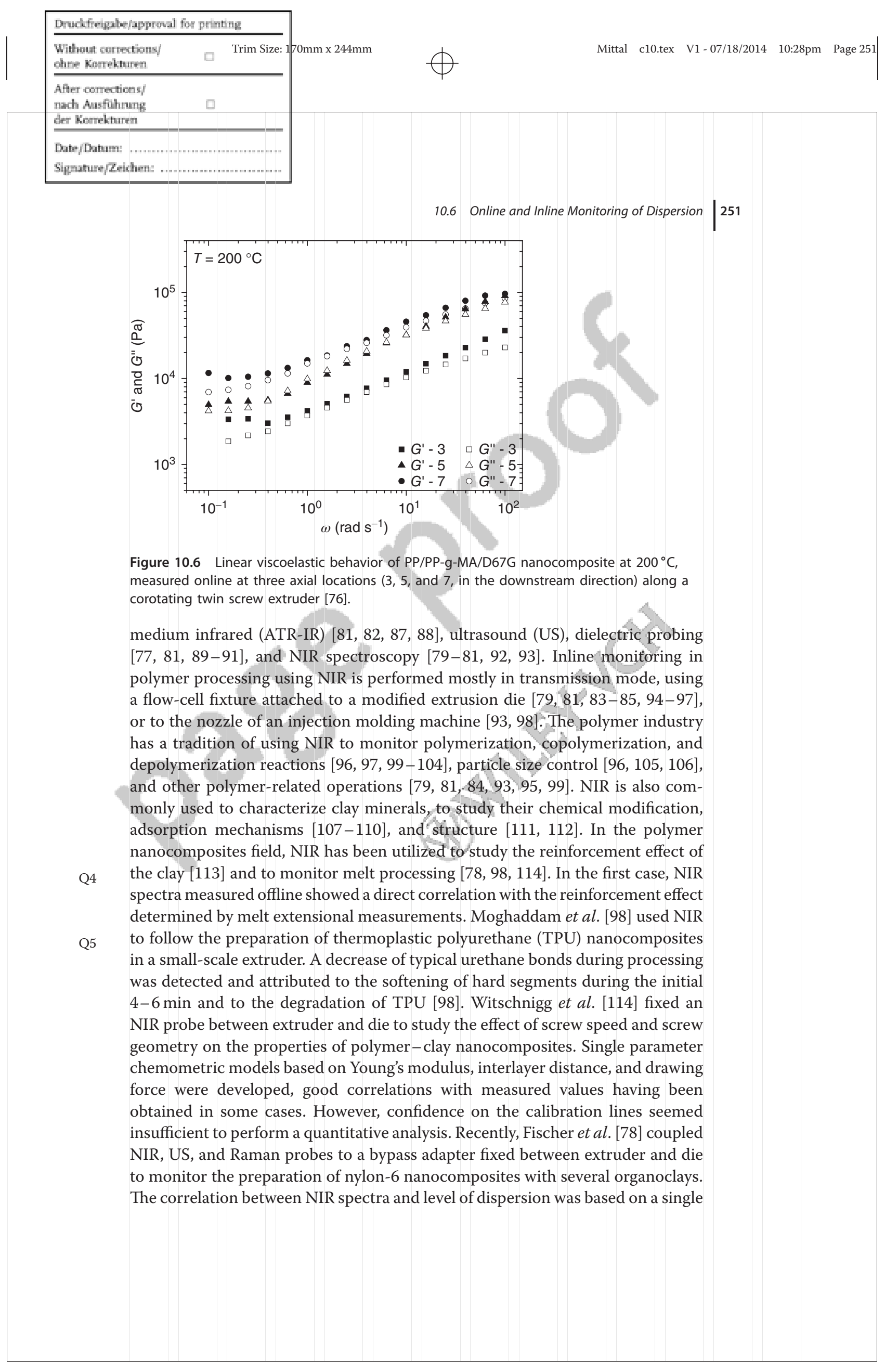

Figure 10.6 Linear viscoelastic behavior of PP/PP-g-MA/D67G nanocomposite at $200^{\circ} \mathrm{C}$, measured online at three axial locations $(3,5$, and 7 , in the downstream direction) along a corotating twin screw extruder [76].

medium infrared (ATR-IR) [81, 82, 87, 88], ultrasound (US), dielectric probing [77, 81, 89-91], and NIR spectroscopy [79-81, 92, 93]. Inline monitoring in polymer processing using NIR is performed mostly in transmission mode, using flow-cell fixture attached to a modified extrusion die [79, 81, 83-85, 94-97], $r$ to the nozzle of an injection molding machine $[93,98]$. The polymer industry has a tradition of using NIR to monitor polymerization, copolymerization, and depolymerization reactions [96, 97, 99-104], particle size control [96, 105, 106], and other polymer-related operations $[79,81,84,93,95,99]$. NIR is also commonly used to characterize clay minerals, to study their chemical modification, dsorption mechanisms [107-110], and structure [111, 112]. In the polymer anocomposites field, NIR has been utilized to study the reinforcement effect of determined by melt extensional measurements. Moghaddam et al. [98] used NIR to follow the preparation of thermoplastic polyurethane (TPU) nanocomposites in a small-scale extruder. A decrease of typical urethane bonds during processing was detected and attributed to the softening of hard segments during the initial 4-6 min and to the degradation of TPU [98]. Witschnigg et al. [114] fixed an NIR probe between extruder and die to study the effect of screw speed and screw geometry on the properties of polymer-clay nanocomposites. Single parameter chemometric models based on Young's modulus, interlayer distance, and drawing force were developed, good correlations with measured values having been btained in some cases. However, confidence on the calibration lines seemed insufficient to perform a quantitative analysis. Recently, Fischer et al. [78] coupled NIR, US, and Raman probes to a bypass adapter fixed between extruder and die to monitor the preparation of nylon-6 nanocomposites with several organoclays. The correlation between NIR spectra and level of dispersion was based on a single 


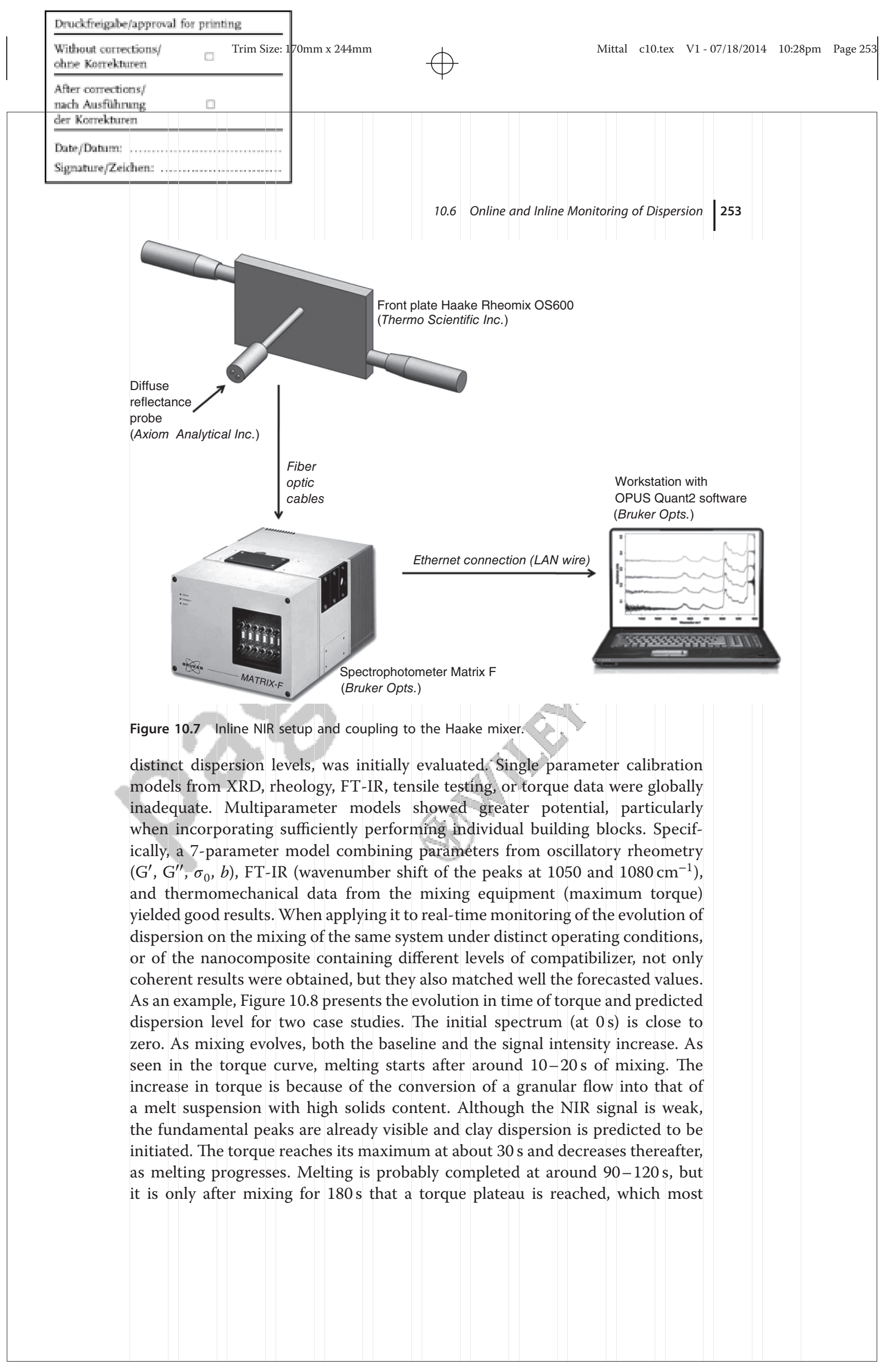




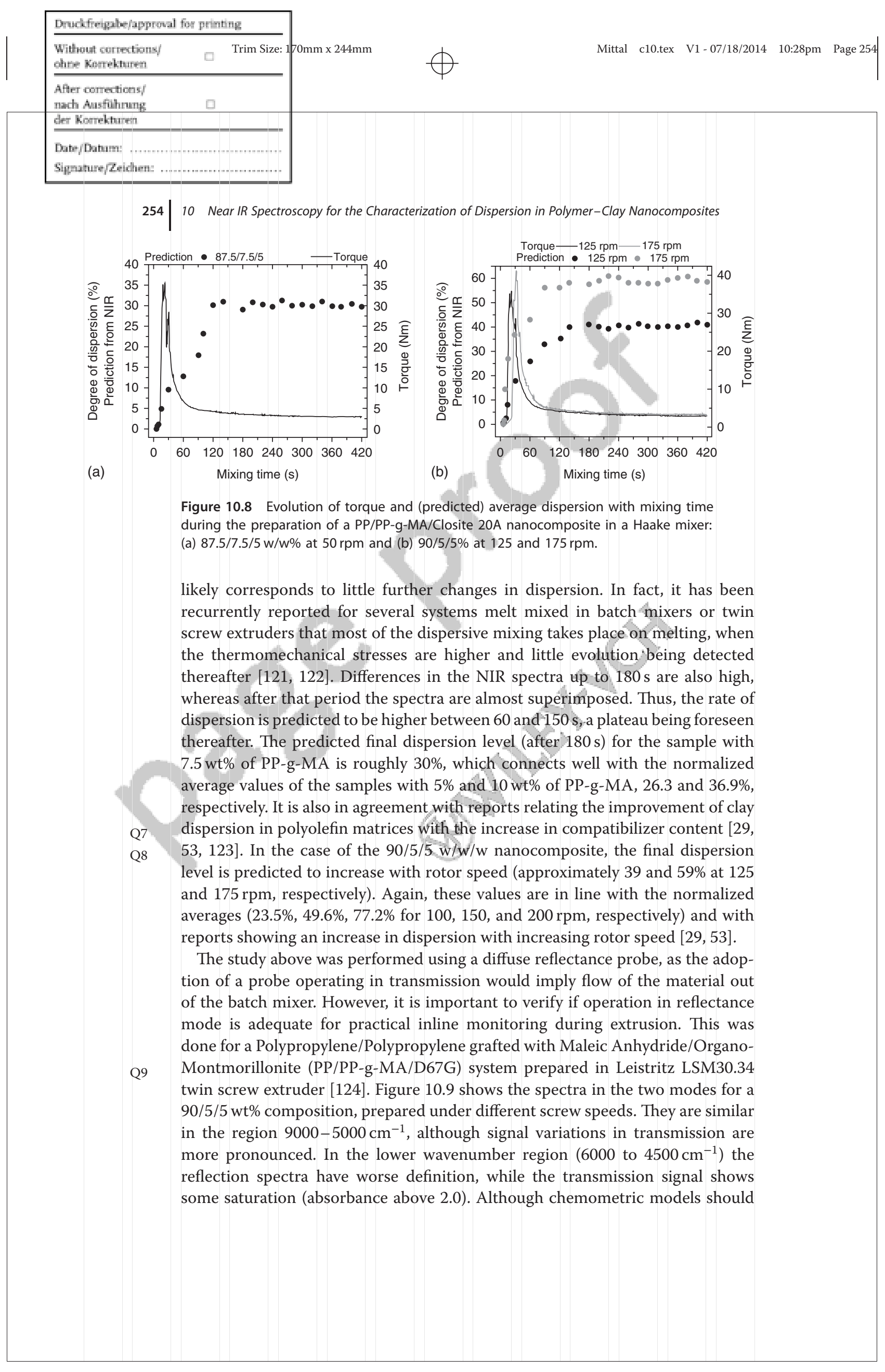

Figure 10.8 Evolution of torque and (predicted) average dispersion with mixing time during the preparation of a PP/PP-g-MA/Closite $20 \mathrm{~A}$ nanocomposite in a Haake mixer:

likely corresponds to little further changes in dispersion. In fact, it has been recurrently reported for several systems melt mixed in batch mixers or twin screw extruders that most of the dispersive mixing takes place on melting, when the thermomechanical stresses are higher and little evolution being detected thereafter $[121,122]$. Differences in the NIR spectra up to $180 \mathrm{~s}$ are also high the rate of dispersion is predicted to be higher between 60 and $150 \mathrm{~s}$, a plateau being foreseen (hereafter. The predicted final dispersion level (after $180 \mathrm{~s}$ ) for the sample with $7.5 \mathrm{wt} \%$ of PP-g-MA is roughly $30 \%$, which connects well with the normalized average values of the samples with $5 \%$ and 10 wt $\%$ of PP-g-MA, 26.3 and $36.9 \%$, respectively. It is also in agreement with reports relating the improvement of clay content $[29$ level is predicted to increase with rotor speed (approximately 39 and 59\% at 125 and $175 \mathrm{rpm}$, respectively). Again, these values are in line with the normalized averages $(23.5 \%, 49.6 \%, 77.2 \%$ for 100,150 , and $200 \mathrm{rpm}$, respectively) and with eports showing an increase in dispersion with increasing rotor speed [29,53].

The study above was performed using a diffuse reflectance probe, as the adopMontmorillonite (PP/PP-g-MA/D67G) system prepared in Leistritz LSM30.34 twin screw extruder [124]. Figure 10.9 shows the spectra in the two modes for a 0/5/5 wt\% composition, prepared under different screw speeds. They are simila in the region $9000-5000 \mathrm{~cm}^{-1}$, although signal variations in transmission are more pronounced. In the lower wavenumber region $\left(6000\right.$ to $\left.4500 \mathrm{~cm}^{-1}\right)$ the reflection spectra have worse definition, while the transmission signal shows some saturation (absorbance above 2.0). Although chemometric models should 


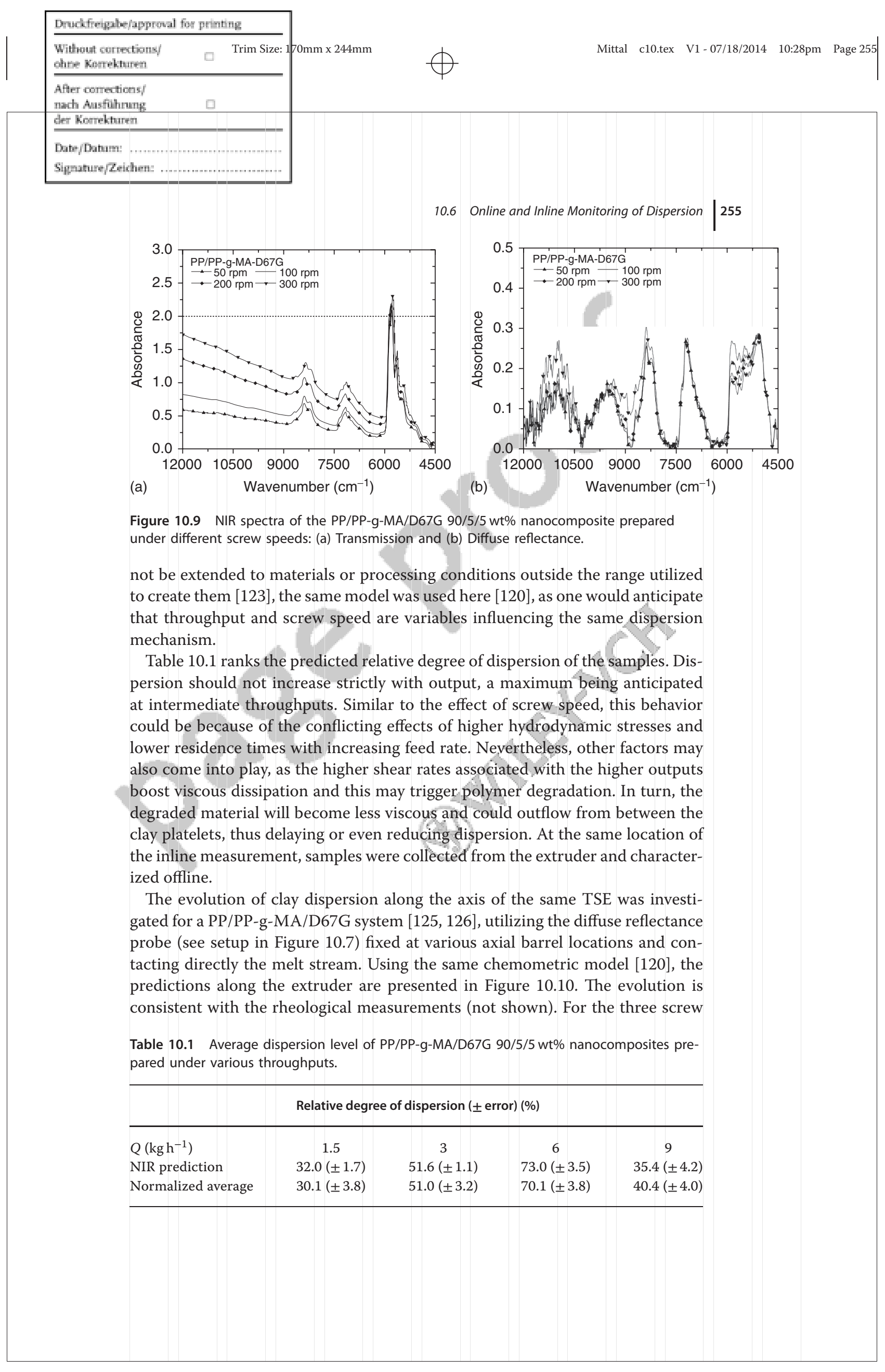




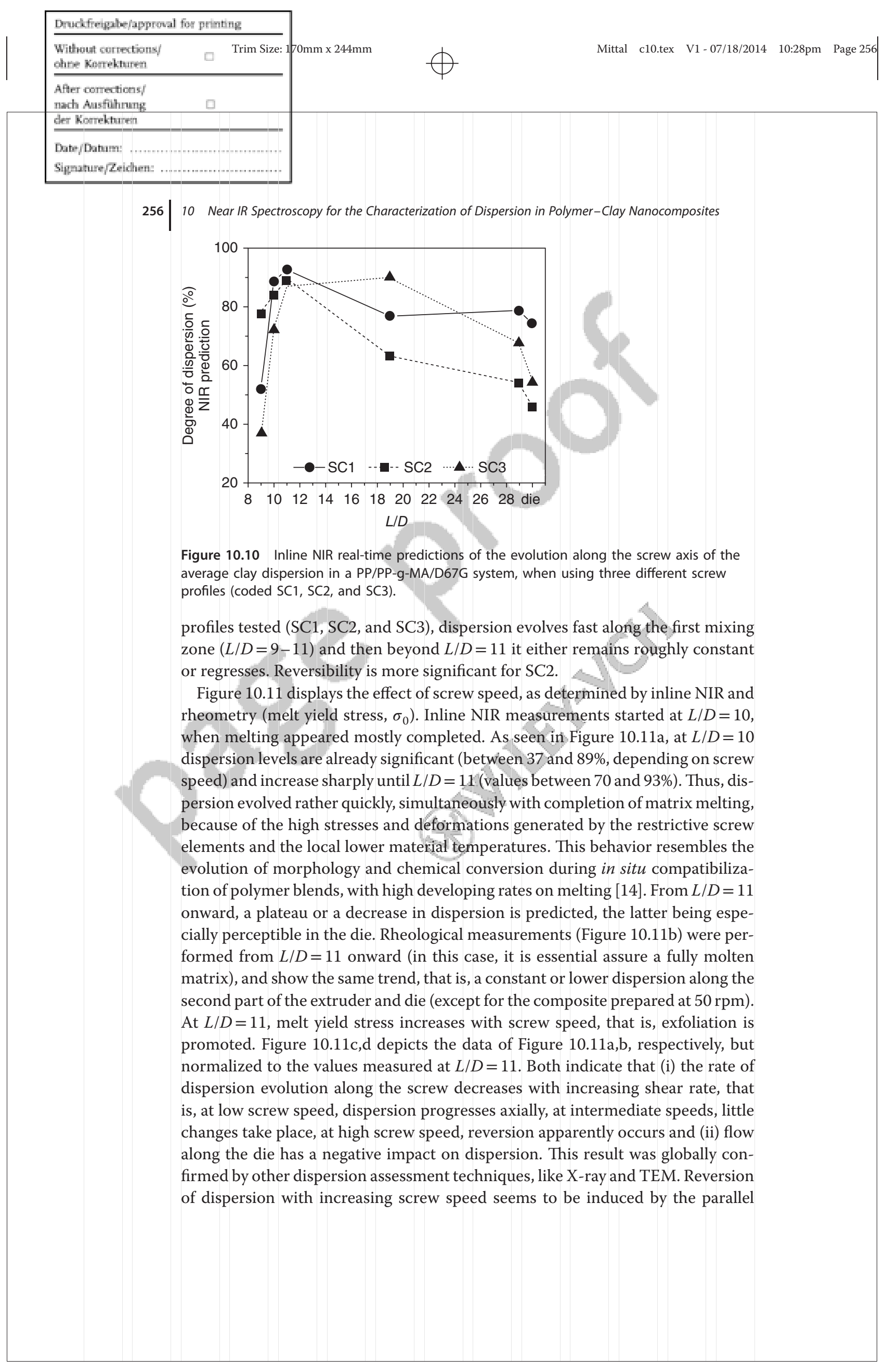

Figure 10.10 Inline NIR real-time predictions of the evolution along the screw axis of the average clay dispersion in a PP/PP-g-MA/D67G system, when using three different screw (coded SC1, SC2, and SC3). regresses. Reversibility is more significant for $\mathrm{SC} 2$. pecause of the high stesses and defornations gera elements and the local lower material temperatures. This behavior resembles the evolution of morphology and chemical conversion during in situ compatibilization of polymer blends, with high developing rates on melting [14]. From $L / D=11$ onward, a plateau or a decrease in dispersion is predicted, the latter being espefromed from $L / D=11$ onward (in this case, it is essential assure a fully molten is, at low screw speed, dispersion progresses axially, at intermediate speeds, little changes take place, at high screw speed, reversion apparently occurs and (ii) flow along the die has a negative impact on dispersion. This result was globally confirmed by other dispersion assessment techniques, like X-ray and TEM. Reversion of dispersion with increasing screw speed seems to be induced by the parallel 


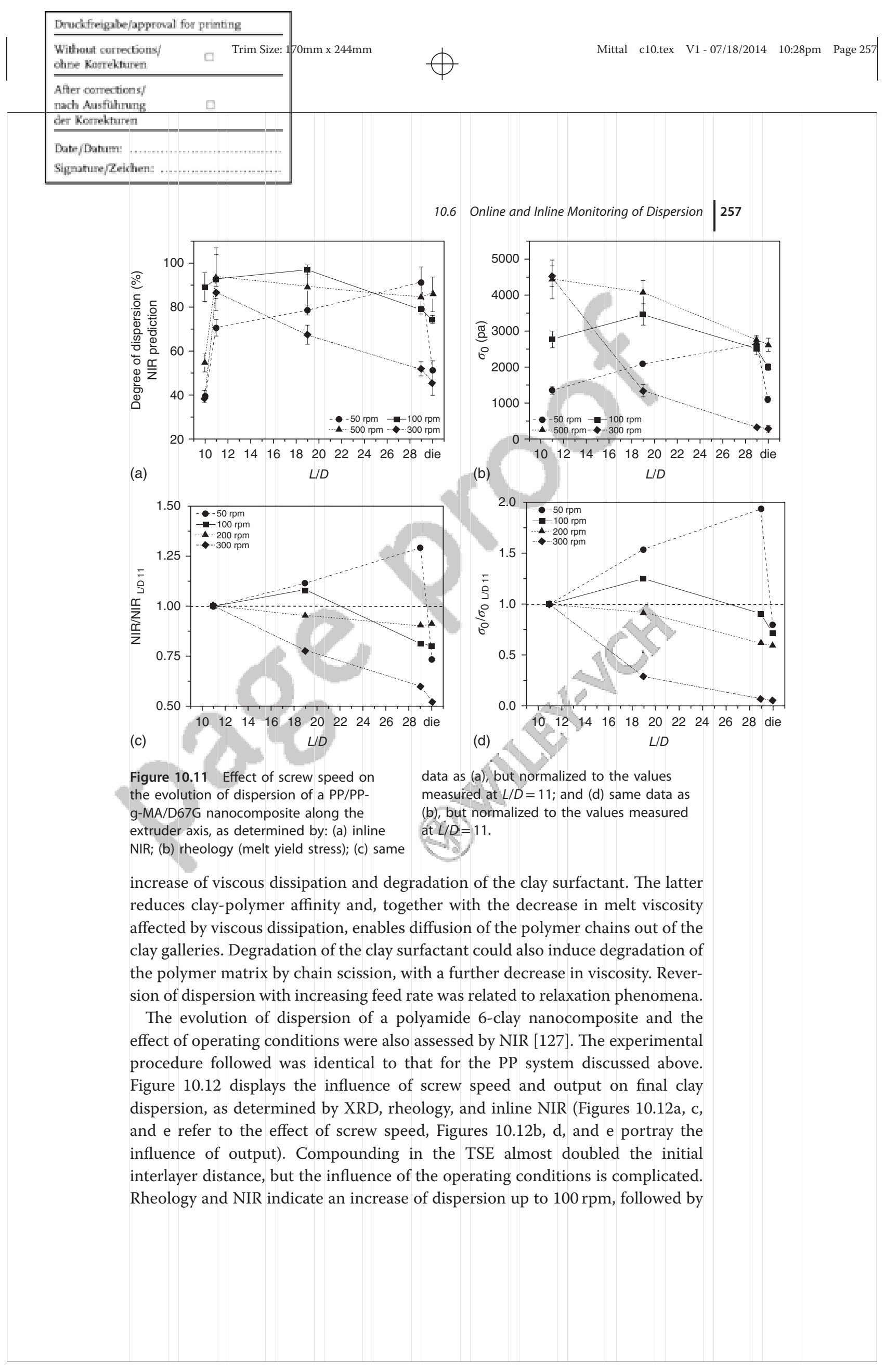




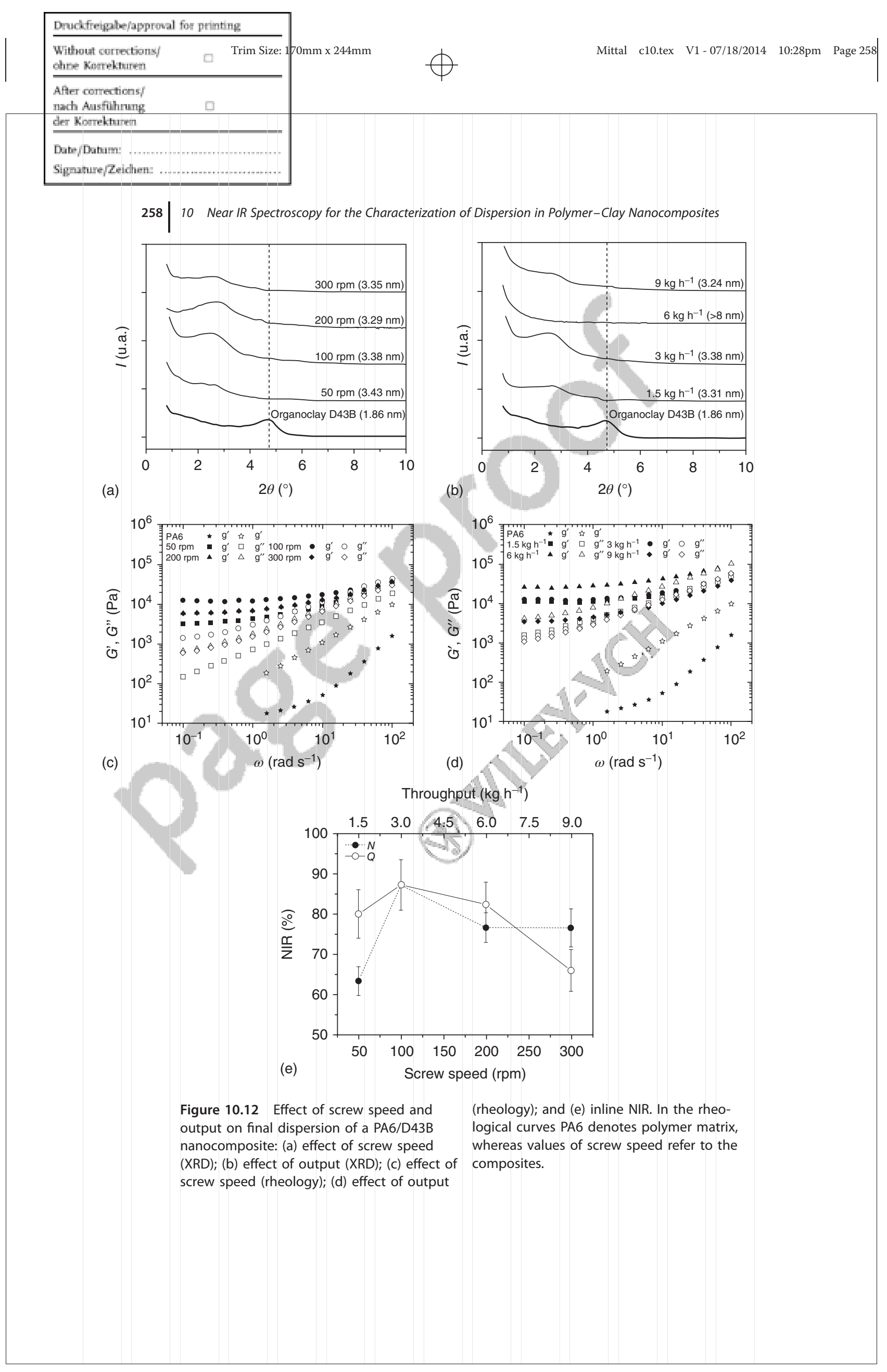




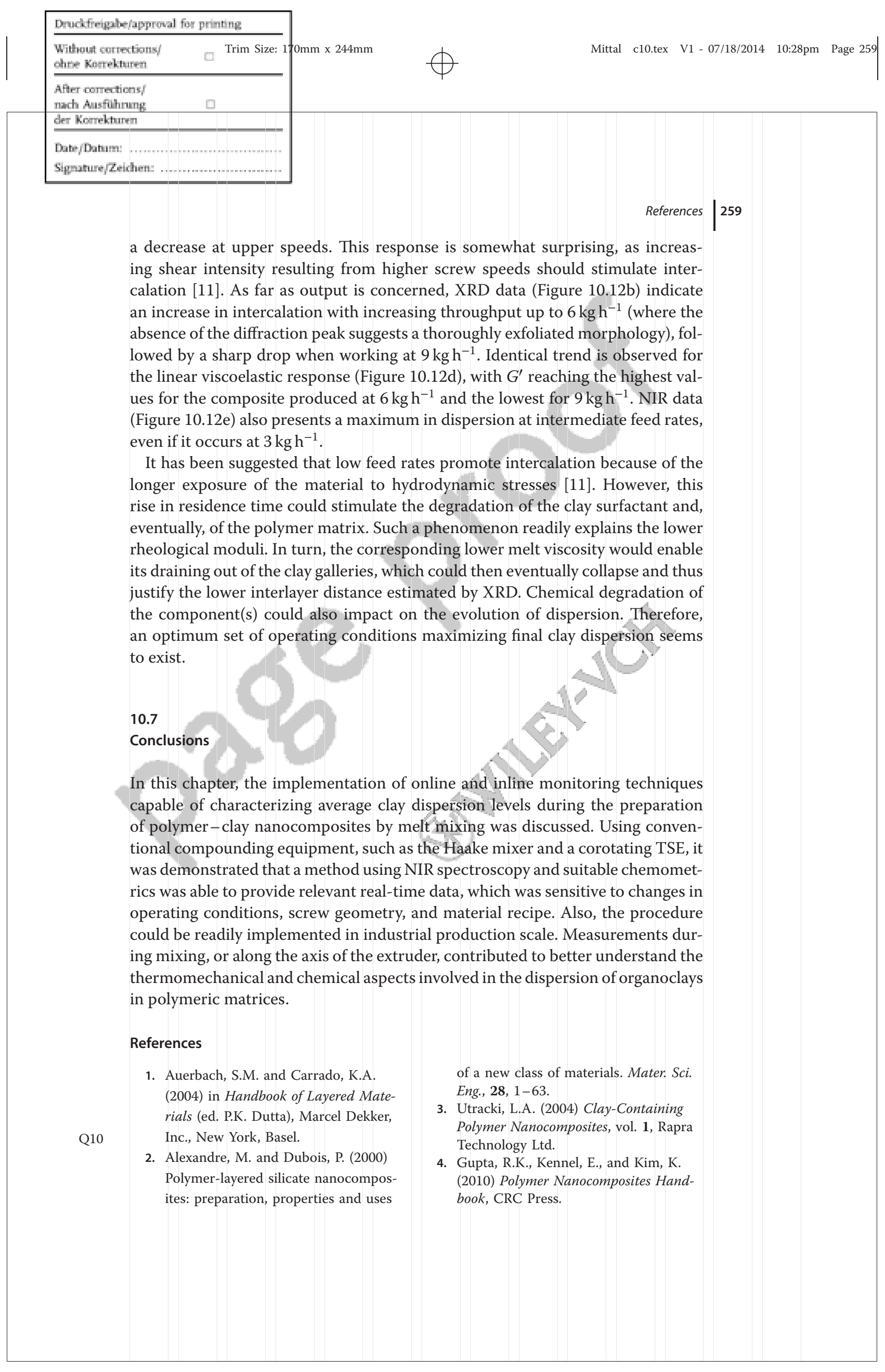

a decrease at upper speeds. This response is somewhat surprising, as increascalation [11]. As far as output is concerned, XRD data (Figure 10.12b) indicate an increase in intercalation with increasing throughput up to $6 \mathrm{~kg} \mathrm{~h}^{-1}$ (where the absence of the diffraction peak suggests a thoroughly exfoliated morphology), followed by a sharp drop when working at $9 \mathrm{~kg} \mathrm{~h}^{-1}$. Identical trend is observed for Pigure 10.12d), with $G^{\prime}$ reachir ues for the composite produced at $6 \mathrm{~kg} \mathrm{~h}^{-1}$ and the lowest for $9 \mathrm{~kg} \mathrm{~h}^{-1}$. NIR data (Figure 10.12e) also presents a maximum in dispersion at intermediate feed rates, even if it occurs at $3 \mathrm{~kg} \mathrm{~h}^{-1}$.

It has been suggested that low feed rates promote intercalation because of the ner exposure of the material to hydrodynamic stresses [11]. However, this an optimum set of operating condit to exist.

10.7

In this chapter, the implementation of online and inline monitoring techniques capable of characterizing average clay dispersion levels during the preparation of polymer - clay nanocomposites by melt mixing was discussed. Using conventional compounding equipment, such as the Haake mixer and a corotating TSE, it was demonstrated that a method using NIR spectroscopy and suitable chemometrics was able to provide relevant real-time data, which was sensitive to changes in operating conditions, screw geometry, and material recipe. Also, the procedure ceadily implemented in industrial production scale. Measurements during mixing, or along the axis of the extruder, contributed to better understand the hermomechanical and chemical aspects involved in the dispersion of organoclays in polymeric matrices.

\section{erences}

Auerbach, S.M. and Carrado, K.A. (2004) in Handbook of Layered Materials (ed. P.K. Dutta), Marcel Dekker,

Alexandre, M. and Dubois, P. (2000) Polymer-layered silicate nanocomposites: preparation, properties and uses of a new class of materials. Mater. Sci. Eng., 28, 1-63. book, CRC Press. 


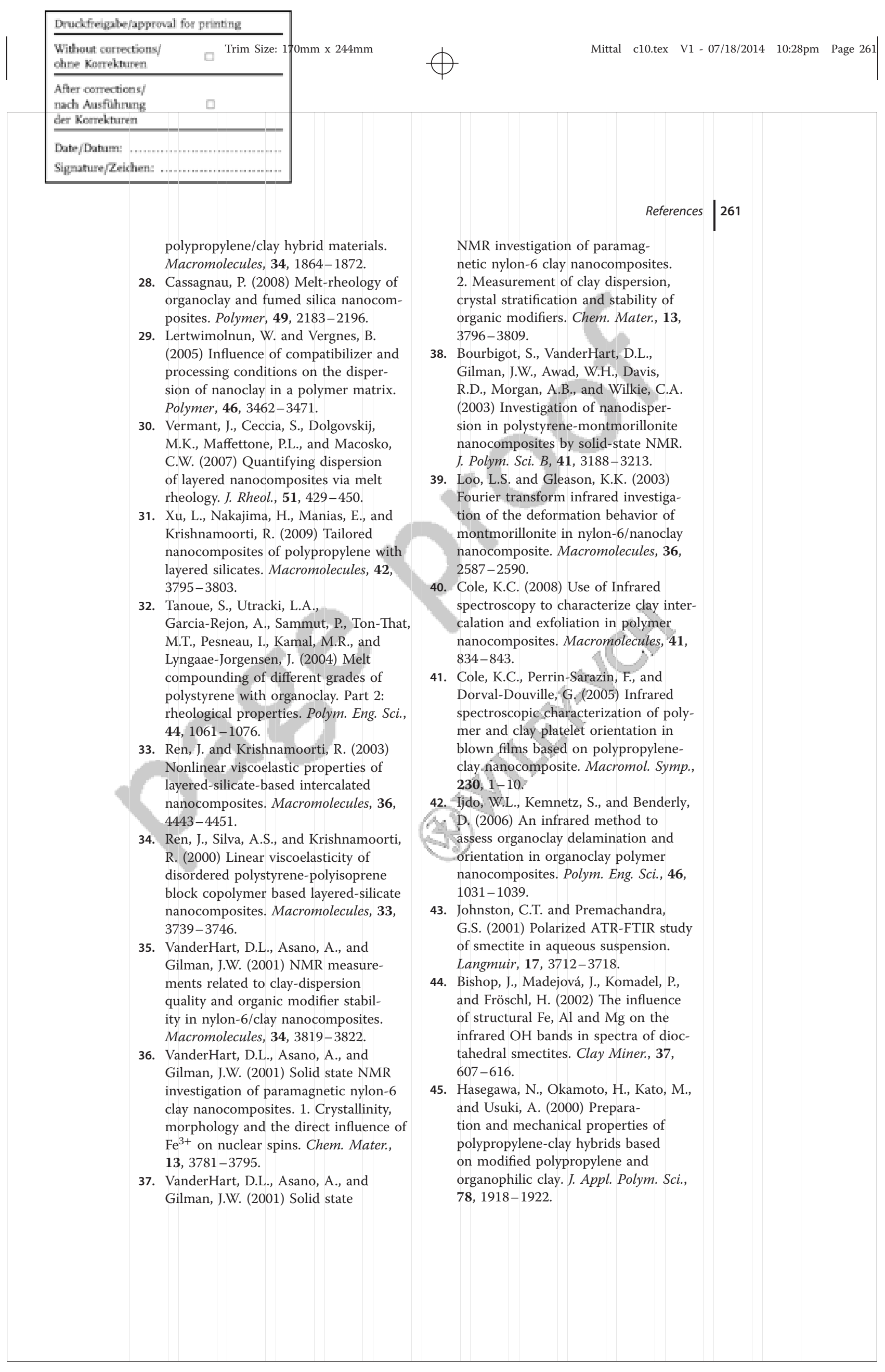




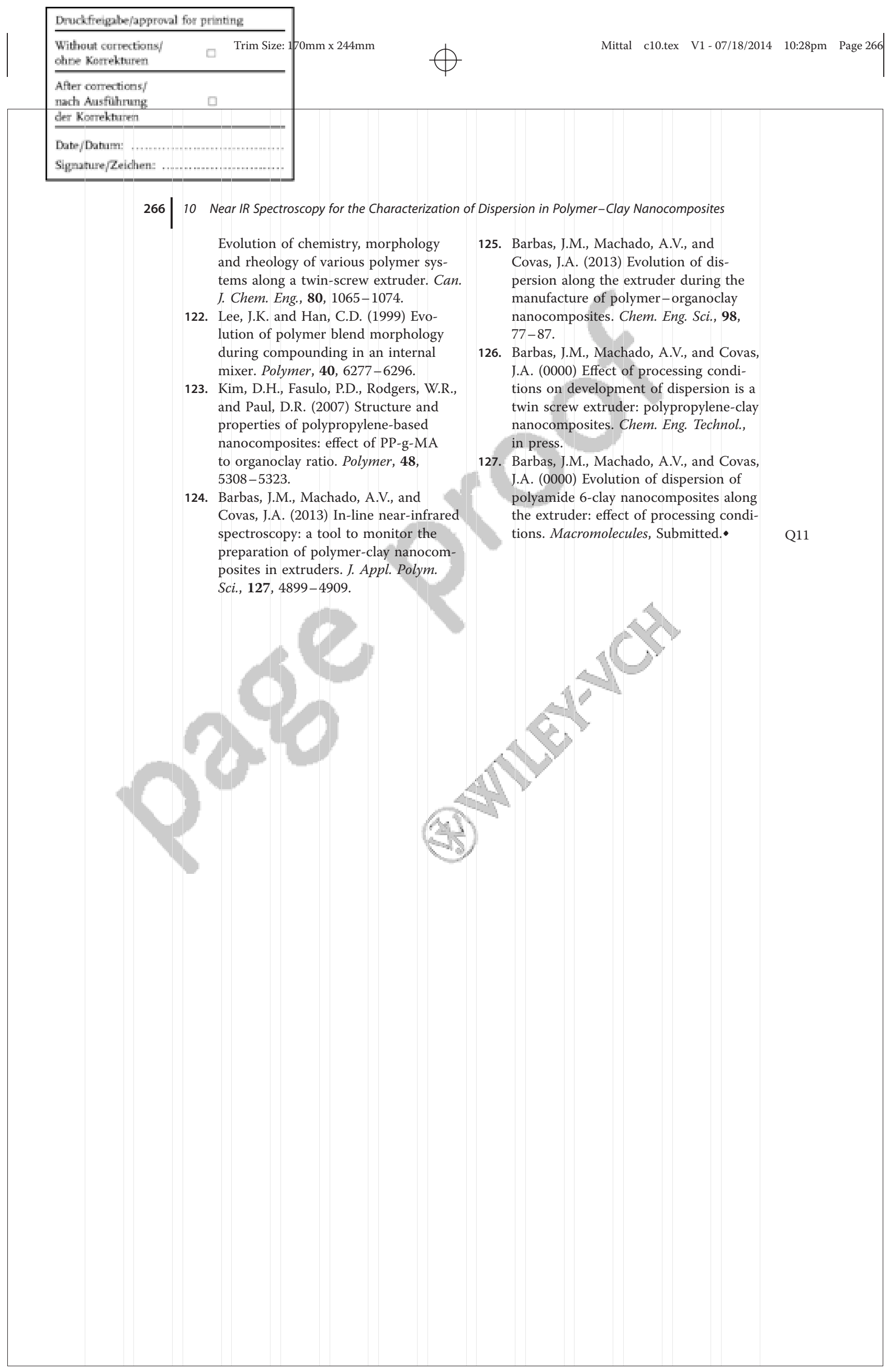




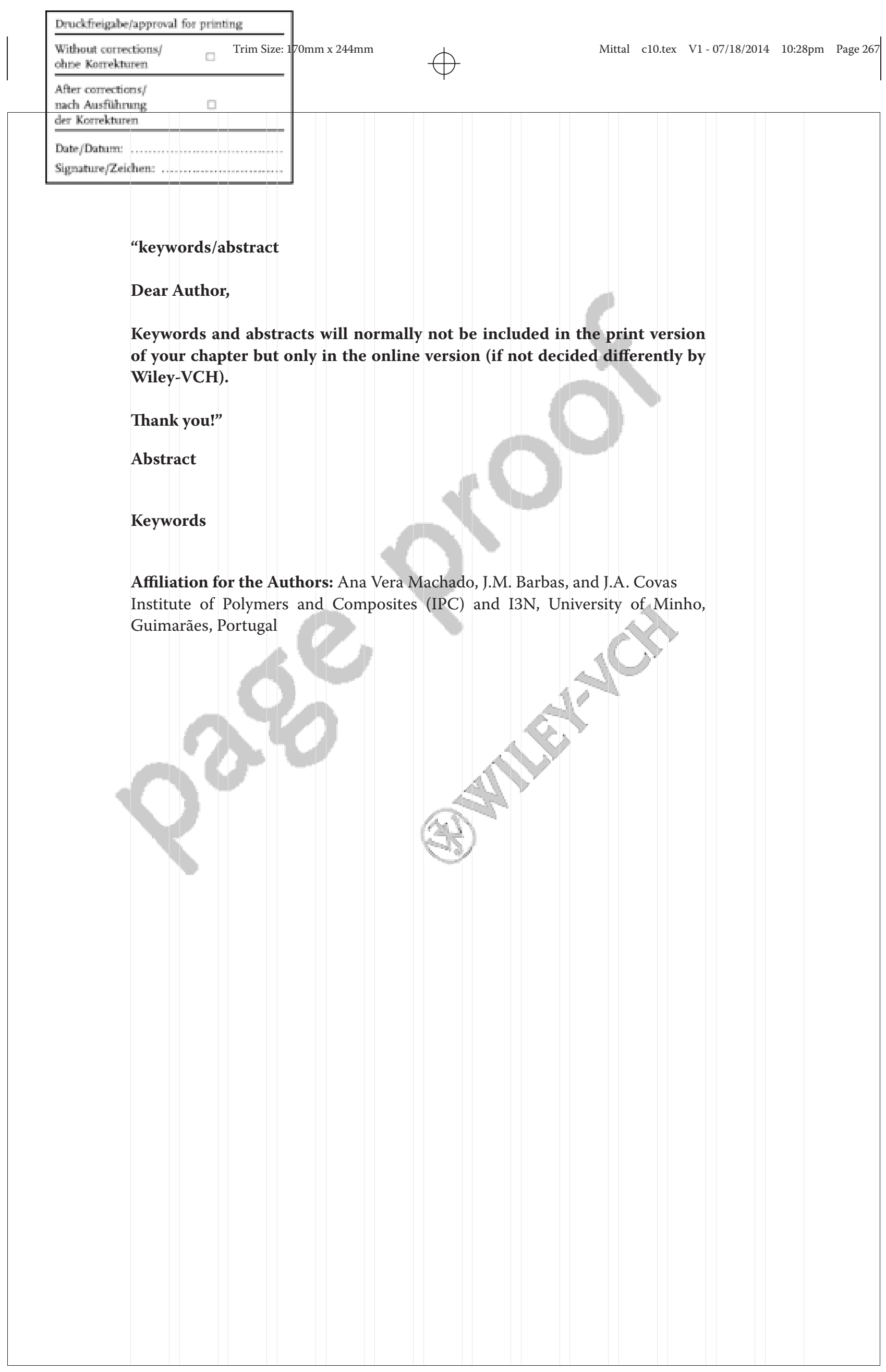




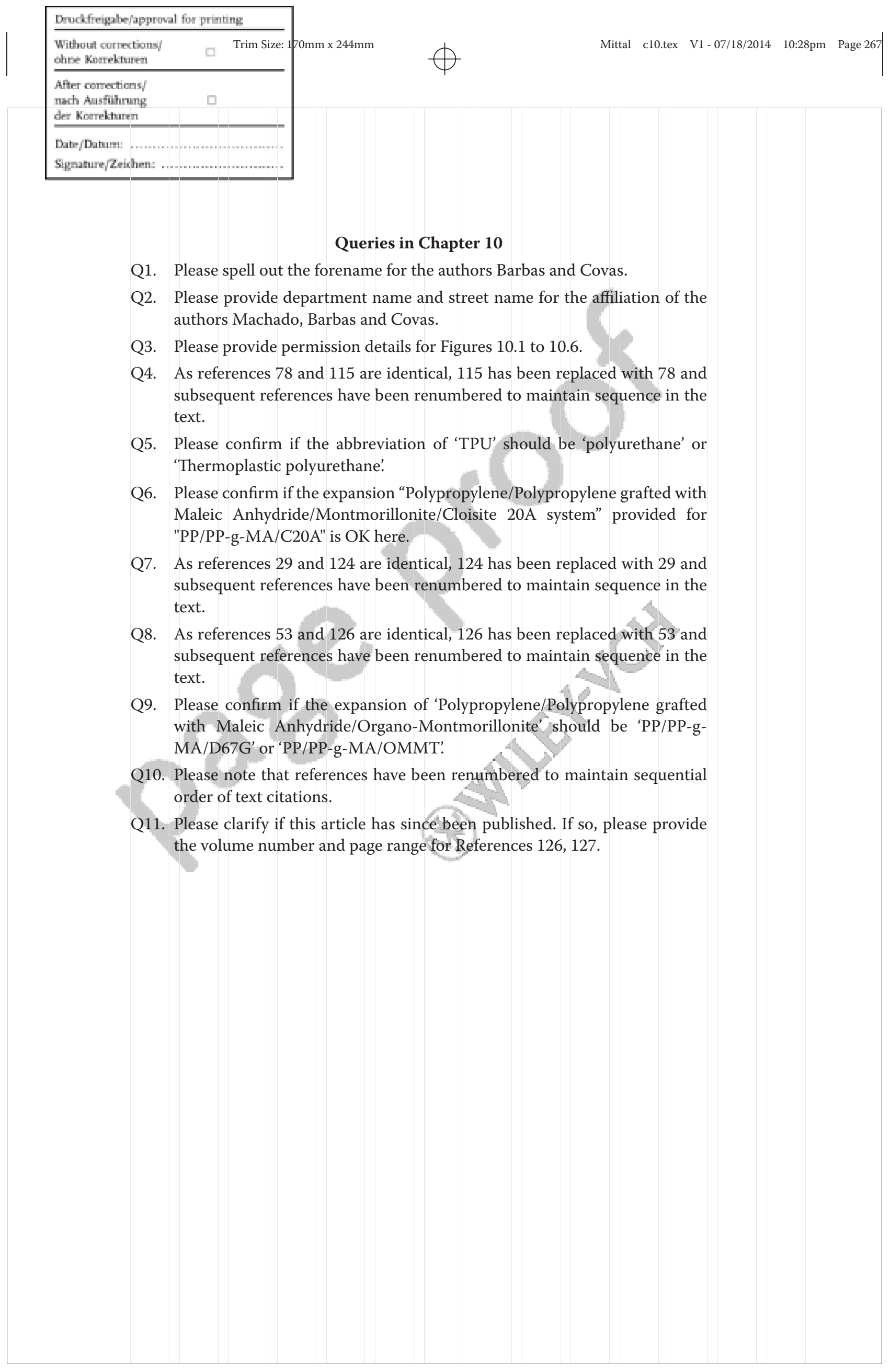

Q1. Please spell out the forename for the authors Barbas and Covas.

Q2. Please provide department name and street name for the affiliation of the authors Machado, Barbas and Covas.

Q4. As references 78 and 115 are identical, 115 has been replaced with 78 and subsequent references have been renumbered to maintain sequence in the text.

Q5. Please confirm if the abbreviation of 'TPU' should be 'polyurethane' or 'Thermoplastic polyurethane'. (20 A stem" provided for As references 29 and 124 are identical, 124 has been replaced with 29 and subsequent references have been renumbered to maintain sequence in the text.

8. As references 53 and 126 are identical, 126 has been replaced with 53 and subsequent references have been renumbered to maintain sequence in the text.

Q9. Please confirm if the expansion of 'Polypropylene/Polypropylene grafted with Maleic Anhydride/Organo-Montmorillonite' should be 'PP/PP-g-

ces have been renumbered to maintain sequential

11. Please clarify if this article has since been published. If so, please provide the volume number and page range for References 126, 127. 